\title{
Size dependence of volume and surface nucleation rates for homogeneous freezing of supercooled water droplets
}

\author{
T. Kuhn ${ }^{1, *}$, M. E. Earle ${ }^{1, * *}$, A. F. Khalizov ${ }^{1, * * *}$, and J. J. Sloan ${ }^{1}$ \\ ${ }^{1}$ Department of Earth and Environmental Sciences, University of Waterloo, Waterloo, ON, Canada \\ *now at: Division of Space Technology, Department of Computer Science, Electrical and Space Engineering, Luleå \\ University of Technology, Kiruna, Sweden \\ ** now at: Cloud Physics and Severe Weather Research Section, Environment Canada, Toronto, ON, Canada \\ *** now at: Department of Atmospheric Sciences, Texas A\&M University, College Station, TX, USA
}

Received: 28 August 2009 - Published in Atmos. Chem. Phys. Discuss.: 28 October 2009

Revised: 10 March 2011 - Accepted: 21 March 2011 - Published: 28 March 2011

\begin{abstract}
The relative roles of volume and surface nucleation were investigated for the homogeneous freezing of pure water droplets. Experiments were carried out in a cryogenic laminar aerosol flow tube using supercooled water aerosols with maximum volume densities at radii between 1 and $3 \mu \mathrm{m}$. Temperature- and size-dependent values of volumeand surface-based homogeneous nucleation rates between 234.8 and $236.2 \mathrm{~K}$ were derived using a microphysical model and aerosol phase compositions and size distributions determined from infrared extinction measurements in the flow tube. The results show that the contribution from nucleation at the droplet surface increases with decreasing droplet radius and dominates over nucleation in the bulk droplet volume for droplets with radii smaller than approximately $5 \mu \mathrm{m}$. This is interpreted in terms of a lowered free energy of ice germ formation in the surface-based process. The implications of surface nucleation for the parameterization of homogeneous ice nucleation in numerical models are considered.
\end{abstract}

\section{Introduction}

The radiative properties of clouds, such as albedo, depend greatly on the concentration, size distribution and phase of cloud particles (Cox, 1971; Lawson et al., 2006). Cirrus clouds, composed of ice crystals, cover about $35 \%$ of the Earth's surface. Owing to their spatial and temporal stability, and interactions with incoming solar radiation and outgoing infrared surface emission, cirrus clouds have a significant in-

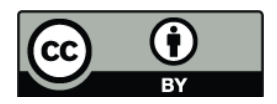

Correspondence to: J. J. Sloan

(sloanj@uwaterloo.ca) fluence on Earth's radiation budget, and hence climate (Liou, 1986). To understand and ultimately predict cloud properties, it is necessary to understand the way in which ice forms in the atmosphere (Cantrell and Heymsfield, 2005).

Below temperatures of about $237 \mathrm{~K}$, cirrus ice is formed primarily by nucleation of ice in aqueous droplets (Heymsfield et al., 2005). Such temperatures are encountered in the upper troposphere, where generally, only few ice-forming particles (ice nuclei) are present. Ice particle concentrations that exceed the number densities of ice nuclei available for heterogeneous nucleation have frequently been reported, providing evidence for the importance of homogeneous nucleation (Heymsfield and Sabin, 1989; Heymsfield and Miloshevich, 1993; Jensen et al., 1998; Rogers et al., 1998; Rosenfeld and Woodley, 2000; Sassen et al., 1985).

The rate of homogeneous nucleation controls the size and concentration of ice particles, and therefore the radiative properties of cirrus clouds, as well as their altitude, spatial extent and lifetime (Heymsfield and Miloshevich, 1993; Jensen and Toon, 1994). Numerical modelling studies (Heymsfield and Sabin, 1989; DeMott et al., 1994, 1997; Jensen et al., 1994) indicate that variations in physical and chemical characteristics of aerosol particle populations and other factors that drive the nucleation of ice lead to the formation of cirrus clouds with different microphysical characteristics. An accurate and detailed understanding of the formation and development of cloud ice crystals is therefore very important.

There are several field and laboratory studies that determined homogeneous nucleation rates for pure water droplets suspended in different media, including air and oils. The laboratory data from studies using airborne water droplets produced nucleation rates whose temperature dependencies

Published by Copernicus Publications on behalf of the European Geosciences Union. 
agree reasonably well (DeMott and Rogers, 1990; Duft and Leisner, 2004; Krämer et al., 1999; Stöckel et al., 2005; Wood et al., 2002). At any given temperature, however, differences of several orders of magnitude in the rate coefficients between different experiments are not uncommon. This may be the result of experimental errors combined with the very strong temperature dependence of freezing rates.

Traditionally, homogeneous nucleation experiments are interpreted using classical nucleation theory, which assumes nucleation rates to be proportional to the volume of water droplets. Therefore, data are usually compared in terms of the volume specific freezing rate coefficient, $J_{\mathrm{V}}$, and results should be independent of the aerosol size distribution used in the experiment. It has been proposed recently that freezing may be favoured on or close to the surface of water droplets (Djikaev et al., 2002; Djikaev, 2008; Tabazadeh et al., 2002). If, rather than forming entirely within the bulk liquid, a crystalline nucleus is attached to the droplet surface such that part of the nucleus' surface is in contact with the surrounding air, then the surface energy of the nucleus is lowered (Djikaev et al., 2002), and so is the free energy of formation. This follows from the condition of partial wetting of an ice crystal with its melt, $\left(\sigma_{\mathrm{i} / \mathrm{v}}-\sigma_{\mathrm{w} / \mathrm{v}}<\sigma_{\mathrm{w} / \mathrm{i}}\right)$. Evaluation of the partial wetting criterion requires knowledge of the surface tensions at the ice-vapour interface, $\sigma_{\mathrm{i} / \mathrm{v}}$, at the liquid water-vapour interface, $\sigma_{\mathrm{w} / \mathrm{v}}$, and at the water-ice interface, $\sigma_{\mathrm{w} / \mathrm{i}}$. The uncertainty of available data on surface tensions (e.g. Pruppacher and Klett, 1997) is, however, too large to evaluate this criterion at typical freezing temperatures with necessary accuracy. The criterion may be met, but the evidence is not strong (Kay et al., 2003).

To describe freezing at the surface, a surface specific rate coefficient, $J_{\mathrm{S}}$, should be used in addition to $J_{\mathrm{V}}$. In this case, the total nucleation rate, $J_{\mathrm{t}}$, for a droplet with radius $r$ would be given by the sum of both surface and volume processes as:

$J_{\mathrm{t}}=J_{\mathrm{V}} \frac{4 \pi}{3} r^{3}+J_{\mathrm{S}} 4 \pi r^{2}$

If it occurs, surface nucleation should be the dominant freezing mechanism for smaller droplets, as can be seen from the radius dependence of volume and surface contributions to the total rate in Eq. (1). Despite considerable previous work, surface nucleation has neither been confirmed, nor ruled out thus far. One way to distinguish between surface and volume freezing is to study the size dependence of the freezing rate coefficients (Djikaev, 2008; Kay et al., 2003), for instance in experiments with water droplets of substantially different sizes. Duft and Leisner (2004) performed such an experiment with 19 and $49 \mu \mathrm{m}$ radius droplets and found the freezing rate to be proportional to the droplet volume. They concluded that volume nucleation dominates at these larger sizes. However, their data would still allow surface nucleation to be important for droplets with radii of $4 \mu \mathrm{m}$ or less, leaving the issue of surface freezing unresolved.
The objective of this study is to carry out size-dependent freezing experiments to investigate the relative roles of volume and surface nucleation in the freezing of micrometersized water droplets, and to determine the corresponding values of $J_{\mathrm{V}}(T)$ and/or $J_{\mathrm{S}}(T)$. This was accomplished using a cryogenic aerosol flow tube with well characterized flow temperature and velocity fields (Khalizov et al., 2006), which provided accurate temperature profiles and a time scale for kinetic measurements. The freezing of aerosol droplets was monitored using Fourier transform infrared (FTIR) spectroscopy, which provided the phase and size distributions of the unfrozen and frozen particles. The technique used to retrieve size distributions does not make any assumptions about the shape of the distributions which could introduce errors and make it difficult to distinguish between surface and volume freezing (Sigurbjörnsson and Signorell, 2008). The experimental freezing data were interpreted within the framework of a microphysical model used previously to determine temperature-dependent volume nucleation rate coefficients (Earle et al., 2010). Here we discuss how the results of experimental measurements can be used to derive important molecular parameters pertaining to the freezing mechanism and distinguish between surface and volume freezing.

\section{Experimental methods}

The experimental method has been described by Earle et al. (2010), and only important points are outlined briefly here. A cryogenic laminar flow tube was used to record infrared (IR) extinction spectra of liquid water and ice aerosols (Khalizov et al., 2006). The flow tube has four independently cooled sections that can be held at different temperatures. The measured temperature variations along a section wall are typically within $\pm 0.5 \mathrm{~K}$. Infrared spectra are recorded by passing a collimated IR beam, modulated by a Michelson interferometer (Bruker Tensor 37), through optical ports in the bottom section of the flow tube, across the aerosol flow. Water vapour spectra recorded at the same temperatures are routinely subtracted from the measured spectra to obtain the aerosol extinction spectra.

To assess the importance of the relative contributions from surface and volume nucleation we studied the size dependence of $J_{\mathrm{V}}$ and $J_{\mathrm{S}}$ using ensembles of water droplets with different initial mean diameters. Millipore ${ }^{\circledR}$ filteredwater was used throughout our study for aerosol generation. Droplets with mean radii (i.e. radii of maximum concentration in volume size distributions) of $1.7 \mu \mathrm{m}$ were generated from pure water by a constant output atomizer (TSI 3076, TSI Inc.). Droplets with larger $(2.9 \mu \mathrm{m})$ and smaller $(1.0 \mu \mathrm{m})$ mean radii were produced by ultrasonic nebulization of pure water and heterogeneous condensation of water vapour on dry sodium chloride $(\mathrm{NaCl})$ nanoparticles, respectively. In the latter case, the $\mathrm{NaCl}$ condensation nuclei were produced by atomizing a dilute aqueous solution 
$\left(1.7 \times 10^{-4} \mathrm{~mol} \mathrm{~L}^{-1}\right)$ of reagent-grade $\mathrm{NaCl}$ (Sigma-Aldrich Co.) and subsequently passing the aqueous droplets through a $60 \mathrm{~cm}$ long Nafion ${ }^{\circledR}$ dryer. The dry salt aerosol was premixed with humidified nitrogen and introduced to the flow tube where the salt particles deliquesced and continued to grow by water vapour uptake.

In the following, the aerosols generated by these three methods - nucleation, atomization and ultrasonic nebulization - are referred to as small, medium and large, respectively. As the aerosol flows through the four flow tube sections, designated A through D (top to bottom), it is exposed to a well-defined temperature profile. In all experiments, sections A and B were maintained at a temperature of $240 \mathrm{~K}$. After conditioning (at the end of section B) the aerosol generated by each method was composed solely of supercooled water droplets. Temperatures of sections $\mathrm{C}$ and $\mathrm{D}$ were varied between 230 and $240 \mathrm{~K}$ (candidate freezing temperature) to induce homogeneous nucleation. At the total flow rate of 10 SLPM, the residence time of aerosols in sections C and D (to the point at which they are observed) is about $35 \mathrm{~s}$.

A retrieval procedure developed in our laboratory was used to determine the phase composition and corresponding size distribution(s) of supercooled water and/or ice particles from their IR spectra (Zasetsky et al., 2004, 2007). The retrieval procedure does not assume any particular shape for the size distributions, such as log-normal; rather, it uses a basis set of 96 reference spectra to iteratively calculate a linear combination that gives the best fit to the measured spectrum. The reference spectra have been computed for spherical monodisperse particles having radii distributed between 0.05 and $11.8 \mu \mathrm{m}$ using Mie scattering code (Bohren and Huffman, 1983) with frequency-dependent complex indices of refraction for supercooled water and ice (Zasetsky et al., 2005).

\section{Model description}

A microphysical model was developed that takes into account both ice nucleation and gas-particle vapour transfer processes to interpret experimentally measured freezing data (Earle et al., 2010). The model uses the measured initial size distribution of conditioned aerosol together with the axial temperature profile and initial guesses of temperaturedependent freezing rate constants and mass accommodation coefficients to compute the evolution of size distributions for water droplets and ice particles. The mass-averaged temperature profile along the flow direction has been determined from a combination of measurements and computational fluid dynamics simulations (Khalizov et al., 2006; Earle et al., 2010). Radial temperature gradients are not considered in the model; previous work has shown that typical temperature differences across the flow tube are less than $0.5 \mathrm{~K}$ at axial positions where most droplets freeze and do not significantly influence the model results (Earle et al., 2010).
The difference between the computed and experimentally retrieved size distributions is minimized by iteratively changing the nucleation rate coefficients and mass accommodation coefficients until the best set of parameters is obtained that reproduce the experimental measurements. The model can be configured to include either a volume-specific nucleation rate coefficient, $J_{\mathrm{V}}$, a surface-specific coefficient, $J_{\mathrm{S}}$, or both. When both volume and surface freezing processes are allowed in the model, the total nucleation rate (per droplet) is given by Eq. (1).

The rate coefficients $J_{\mathrm{V}}$ and $J_{\mathrm{S}}$ are parameterized as follows (Tabazadeh et al., 2002; Turnbull and Fisher, 1949):

$$
\begin{gathered}
J_{\mathrm{V}}=\frac{N_{\mathrm{V}} k T}{h} \exp \left(-\frac{A_{\mathrm{V}}-B_{\mathrm{V}} T}{k T}\right) \\
J_{\mathrm{S}}=\frac{N_{\mathrm{S}} k T}{h} \exp \left(-\frac{A_{\mathrm{S}}-B_{\mathrm{S}} T}{k T}\right)
\end{gathered}
$$

where $N_{\mathrm{V}}=3.35 \times 10^{22} \mathrm{~cm}^{-3}$ is the number concentration of water molecules in liquid water and $N_{\mathrm{S}}=N_{\mathrm{V}}^{2 / 3}$ is the number concentration of water molecules in the surface layer. The parameters $A_{\mathrm{V} / \mathrm{S}}$ and $B_{\mathrm{V} / \mathrm{S}}$ are not varied directly in the iterative fitting procedure of the model, but are first transformed to the parameters $a_{\mathrm{V} / \mathrm{S}}$ and $b_{\mathrm{V} / \mathrm{S}}$, which correspond to the inclination (slope) and intercept of the exponentially-changing nucleation rate coefficients when plotted on a $\log J-T$ plot (Earle et al., 2010).

Equation (1) can be written as:

$$
J_{\mathrm{t}}=J_{\mathrm{V}} \frac{4 \pi}{3} r^{3}\left(1+\frac{3 J_{\mathrm{S}}}{r J_{\mathrm{V}}}\right)
$$

which shows that the contribution of surface freezing relative to volume freezing is proportional to $r^{-1}$. Hence, surface freezing should be important for smaller radii and dominant below a certain critical radius, $r_{\mathrm{c}}$ (Krämer et al., 1999). If we define this critical radius as the size at which surface and volume freezing are equally important, then from Eq. (4):

$r_{\mathrm{c}}=\frac{3 J_{\mathrm{S}}}{J_{\mathrm{V}}}$

Thus, by using freezing experiments conducted with aerosols having different mean radii, we can evaluate $r_{\mathrm{c}}$ and the importance of surface freezing as a function of radius as given by Eq. (4). The model can then determine values of $J_{\mathrm{V}}$ and $J_{\mathrm{S}}$ (from the best fit) using Eq. (4). In the following sections, we describe the analysis of experiments performed with three different initial aerosol size distributions to derive the surface and volume nucleation rate coefficients, and thereby assess the size dependence of the respective nucleation processes. 
Table 1. Parameters resulting from separate model fits to small, medium and large aerosols, assuming only volume or surface nucleation. The quality of fit parameter $\chi$ has been defined by Earle et al. (2010).

\begin{tabular}{lccc}
\hline & $\chi$ & $A_{V}$ or $A_{S}(\mathrm{~J})$ & $B_{V}$ or $B_{S}\left(\mathrm{~J} \mathrm{~K}^{-1}\right)$ \\
\hline Small, $J_{\mathrm{V}}$ & $3.65 \times 10^{-3}$ & $-2.57720 \times 10^{-18}$ & $-1.18023 \times 10^{-20}$ \\
Small, $J_{\mathrm{S}}$ & $3.56 \times 10^{-3}$ & $-2.50010 \times 10^{-18}$ & $-1.13796 \times 10^{-20}$ \\
Med., $J_{\mathrm{V}}$ & $3.51 \times 10^{-3}$ & $-2.53889 \times 10^{-18}$ & $-1.16432 \times 10^{-20}$ \\
Med., $J_{\mathrm{S}}$ & $3.83 \times 10^{-3}$ & $-2.27879 \times 10^{-18}$ & $-1.04364 \times 10^{-20}$ \\
Large, $J_{\mathrm{V}}$ & $2.16 \times 10^{-3}$ & $-2.40898 \times 10^{-18}$ & $-1.10989 \times 10^{-20}$ \\
Large, $J_{\mathrm{S}}$ & $2.49 \times 10^{-3}$ & $-2.11421 \times 10^{-18}$ & $-0.97384 \times 10^{-20}$ \\
\hline
\end{tabular}

\section{Results}

The model was used to fit the measured results for three separate groups of experiments, each corresponding to one aerosol size - small ( 3 experiments at different candidate freezing temperatures), medium (5 experiments), and large (3 experiments) - having maxima in their volume size distributions at radii of $1.0,1.7$, and $2.9 \mu \mathrm{m}$, respectively. The size distributions of these supercooled liquid water aerosols were determined with all four flow tube sections set at $240 \mathrm{~K}$, as reported by Earle et al. (2010). They were then used as input for the microphysical model, i.e. the initial supercooled water distributions in freezing experiments.

Initially, separate model runs were performed by assuming that freezing is only a surface process (using Eq. (1) and setting $\left.J_{\mathrm{V}}=0\right)$, yielding a value of $J_{\mathrm{S}}$, or alternatively, only a volume process $\left(J_{S}=0\right.$ in Eq. 1$)$, which yields a value of $J_{\mathrm{V}}$. Model runs assuming only a volume process using the same aerosol sizes (small, medium and large) have been carried out by Earle et al. (2010). They showed that when classical, volume-based nucleation was assumed in the model, an unexpected size dependence was observed, in which $J_{V}$ increased with decreasing aerosol size. Values of the mass accommodation coefficients, $\alpha_{\mathrm{w}}$ and $\alpha_{\text {ice }}$, which are required to compute droplet evaporation and ice growth by deposition, were determined from the model in the same study. Here we use the reported average values and keep these fixed during model runs to reduce the number of unknown parameters during the fitting process. The values used are $\alpha_{\mathrm{w}}=0.054$ and $\alpha_{\text {ice }}=0.031$.

Figure 1 shows the resulting nucleation rate coefficients $J_{\mathrm{S}}$ (dashed lines) and $J_{\mathrm{V}}$ (solid lines) assuming either surface or volume nucleation, respectively. For all experiments, the model results indicate that the freezing events occur almost exclusively in a narrow range of temperatures $(\approx 0.25 \mathrm{~K})$ near the minimum temperature reached in the flow tube (Earle et al., 2010). Therefore, for each group of experiments, Fig. 1 shows the rate coefficients over a temperature range that spans the minima of the corresponding experiments and extends $0.25 \mathrm{~K}$ towards warmer temperatures. For the small,

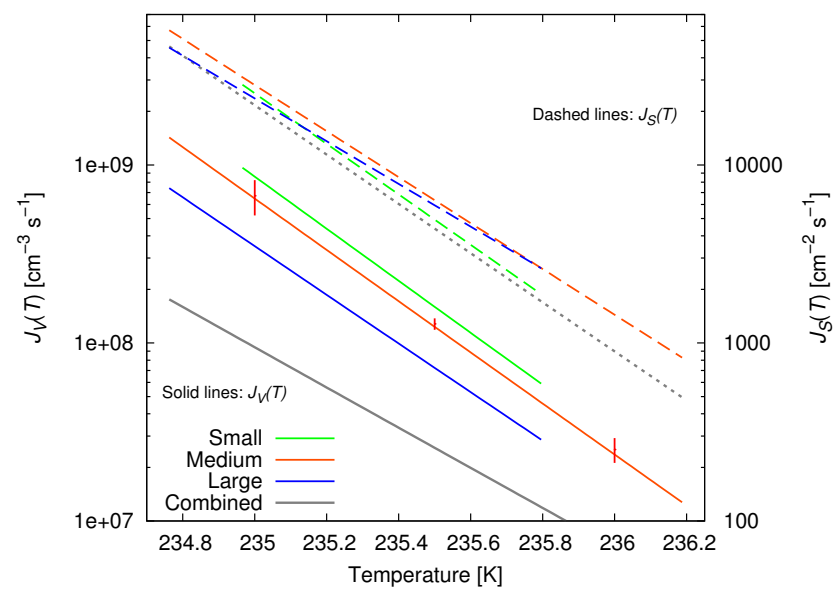

Fig. 1. Volume- and surface-specific nucleation rate coefficients resulting from separate model fits to small, medium and large aerosols. Results from a model run which included contributions from volume and surface nucleation simultaneously, and combined all experiments from small, medium and large aerosols, are shown as grey lines. Error bars are shown for volume-specific nucleation rate coefficients at different temperatures. The temperature ranges used in each model run are discussed in the text.

medium and large aerosols, these ranges are between 235.0 to $235.8 \mathrm{~K}, 234.8$ and $236.2 \mathrm{~K}$ and 234.8 to $235.8 \mathrm{~K}$ respectively. For each size group, the data can be fitted equally well by using only $J_{\mathrm{V}}$ or only $J_{\mathrm{S}}$ in the model, as can be judged from Table 1, which shows the average values of $\chi$, the quality of fit parameter introduced by Earle et al. (2010). Uncertainties in the rate coefficients are indicated as error bars, which are shown at different temperatures in Fig. 1. The method of determining these uncertainties for modeldetermined nucleation rate coefficients has been discussed by Earle et al. (2010).

If freezing is predominantly a volume process, then the size dependence of the rate should scale with the droplet volume (i.e. with $r^{3}$ ) and the volume-specific rate coefficient $J_{\mathrm{V}}$ (for a given temperature) should be invariant for experiments with different aerosol sizes. This is not the case for our data. On the contrary, $J_{\mathrm{V}}$ shows a definite trend with aerosol size, becoming greater with decreasing droplet radius. Our results for $J_{\mathrm{S}}$, however, are size independent, showing that the rate is proportional to the droplet surface area $\left(r^{2}\right)$ in the size range of our experiments and thus a mechanism involving surface nucleation describes the freezing process best.

We conducted additional calculations using the three size groups combined into one data set with either volume or surface freezing mechanism. The quality of the fit was better when using the assumption that nucleation occurs at the surface $\left(\chi \approx 3.7 \times 10^{-3}\right)$ rather than in the volume of the water droplets $\left(\chi \approx 5.3 \times 10^{-3}\right)$, providing clear evidence for the importance of surface freezing. One may arrive at the same conclusion by considering the spread in $J_{\mathrm{V}}$ for different sizes 


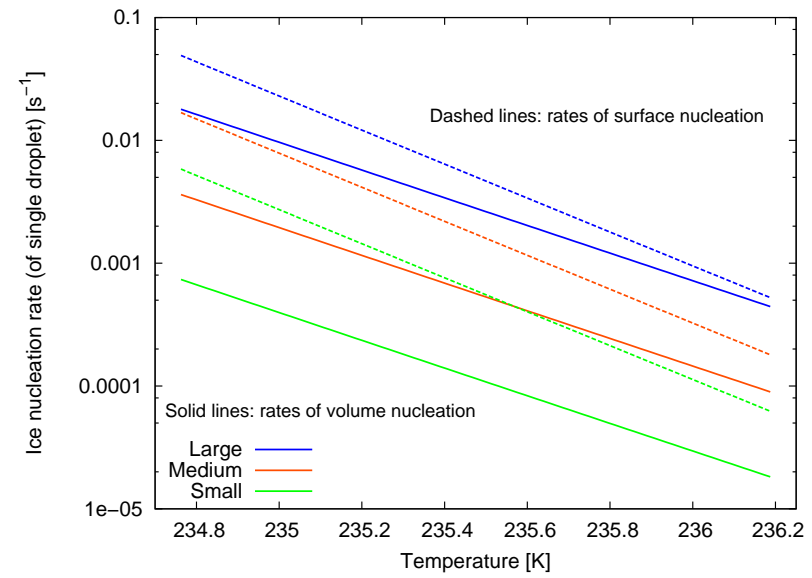

Fig. 2. Nucleation rates resulting from fits including both volume and surface freezing. Contributions to nucleation rates from volume and surface processes are shown separately for each of the three droplet sizes. For each size, the surface nucleation rate is greater than the volume rate. This difference increases with decreasing droplet size, as expected from the different radius dependence of surface and volume nucleation rates.

(solid lines) in Fig. 1, which in the case of a combined size calculation must be fitted with only one $J_{\mathrm{V}}(T)$ curve.

In a final test, we used Eq. (4) for the total nucleation rate in the model, thereby including contributions from both the volume and surface processes simultaneously (still using the combined data set comprising all three aerosol sizes). The resulting fits yielded temperature-dependent values of both $J_{\mathrm{V}}$ and $J_{\mathrm{S}}$. The quality of fit parameter $\chi$ was now $\approx 3.5 \times 10^{-3}$. The results, given by the fit parameters, are summarized in Table 2. The coefficient $J_{\mathrm{S}}(T)$ from this combined volume and surface model run (grey dashed line in Fig. 1) is very similar to the three curves of $J_{\mathrm{S}}$ from fits using only surface freezing for the different aerosols shown in Fig. 1 (dashed lines). The coefficient $J_{\mathrm{V}}(T)$ from the combined run (grey solid line), on the other hand, is approximately three times smaller than the lowest curve of $J_{\mathrm{V}}$ from fits using only volume freezing (solid lines). This indicates that surface freezing dominates over volume freezing if both are included in the model at the same time. This is also depicted in Fig. 2, which shows the nucleation rates derived from the coefficients $J_{\mathrm{V}}$ and $J_{\mathrm{S}}$ (from combined run) for each size. Contributions from volume and surface nucleation, calculated as $J_{\mathrm{V}}(4 \pi / 3) r^{3}$ and $J_{\mathrm{S}} 4 \pi r^{2}$, respectively, are shown separately (as solid and dashed lines) and are generated using the three radii $1.0,1.7$ and $2.9 \mu \mathrm{m}$ representing the small, medium and large aerosols. The significance of surface freezing (with respect to the volume freezing) increases with decreasing aerosol size, as would be expected from the different radius dependences of surface and volume freezing rates (Eqs. 1 and $4)$.

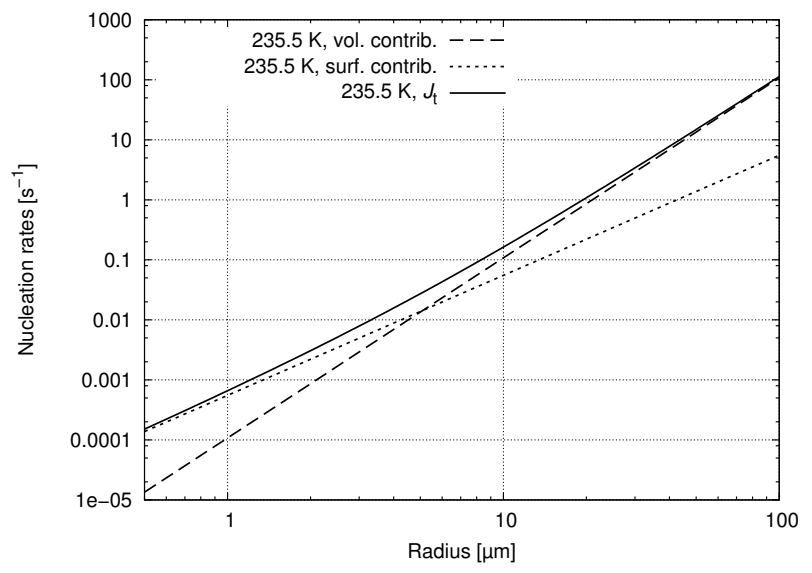

Fig. 3. Total nucleation rate for one droplet as a function of its radius. Contributions from freezing events in the bulk volume or at the surface are shown as dashed and dotted lines which intersect at $r_{\mathrm{c}}$. For droplets with radii smaller than $r_{\mathrm{c}}$ nucleation at the surface is more probable. The sum of these contributions is the total rate, $J_{\mathrm{t}}$.

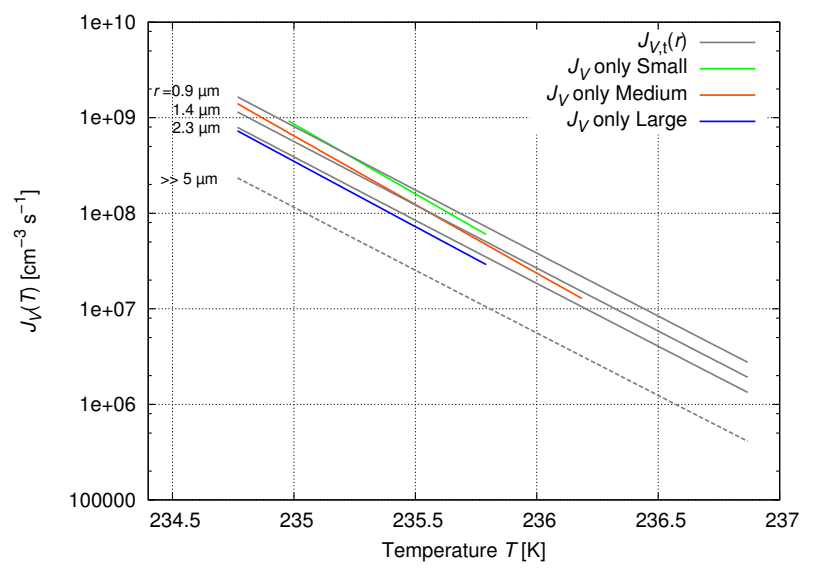

Fig. 4. Results for $J_{\mathrm{V}}$ (assuming volume only freezing, cfr. Fig. 1) compared with volume-specific total nucleation rate coefficients $J_{\mathrm{V}, \mathrm{t}}$ determined from fits assuming both volume and surface nucleation. The surface contribution is incorporated into the volumespecific rate coefficient $J_{\mathrm{V}, \mathrm{t}}$, which therefore becomes size dependent. The $J_{\mathrm{V}, \mathrm{t}}$ curves corresponding to the three aerosol sizes agree well with the $J_{\mathrm{V}}$ curves, as expected. Note that instead of the mean radii $(1.0,1.7$ and $2.9 \mu \mathrm{m})$, the radii of spheres with average volume have been used for this comparison. These radii are $0.9,1.4$, and $2.3 \mu \mathrm{m}$ for the small, medium and large aerosols. The limiting case of large droplets is also shown (labeled as " $r \gg 5 \mu \mathrm{m}$ ").

Figure 3 shows nucleation rates as a function of droplet radius calculated from Eq. (1). It illustrates that for a given temperature $(235.5 \mathrm{~K})$ volume nucleation predominates for droplets having larger radii whereas surface nucleation becomes dominant for smaller droplets. The radius $r_{\mathrm{c}}$ given by Eq. (5), where both volume and surface process contribute 
Table 2. Parameters from the best simultaneous fit to results from a group of experiments with small, medium and large aerosols using the microphysical model with freezing by volume and/or surface nucleation. The last row contains data discussed in Sect. 5 .

\begin{tabular}{lccccc}
\hline & $\chi$ & $A_{\mathrm{V}}(\mathrm{J})$ & $B_{\mathrm{V}}\left(\mathrm{J} \mathrm{K}^{-1}\right)$ & $A_{\mathrm{S}}(\mathrm{J})$ & $B_{\mathrm{S}}\left(\mathrm{J} \mathrm{K}^{-1}\right)$ \\
\hline Volume & $5.27 \times 10^{-3}$ & $-2.54864 \times 10^{-18}$ & $-1.16851 \times 10^{-20}$ & & \\
Surface & $3.67 \times 10^{-3}$ & & & $-2.29544 \times 10^{-18}$ & $-1.05086 \times 10^{-20}$ \\
Eq. (4) & $3.53 \times 10^{-3}$ & $-1.99084 \times 10^{-18}$ & $-0.93376 \times 10^{-20}$ & $-2.44194 \times 10^{-18}$ & $-1.11342 \times 10^{-20}$ \\
Eq. (8) & $3.54 \times 10^{-3}$ & $-2.32316 \times 10^{-18}$ & $-1.07490 \times 10^{-21}$ & & \\
\hline
\end{tabular}

equally to $J_{\mathrm{t}}$, is at $5.1 \mu \mathrm{m}$ for the case shown in Fig. 3. It varies from $7.7 \mu \mathrm{m}$ to $3.4 \mu \mathrm{m}$ over the range of freezing temperatures encountered in our experiments $\left(r_{\mathrm{c}}\right.$ decreases with increasing temperature, as can be judged from the slightly different temperature trends of the grey lines representing $J_{\mathrm{V}}$ and $J_{\mathrm{S}}$ in Fig. 1). This supports our earlier conclusion (based on independent calculations for each freezing mechanism) that the droplet size range in our experiments extends to sufficiently small radii that $J_{\mathrm{S}}$ dominates the nucleation process. It should also be noted that our large aerosol size includes radii up to about $5 \mu \mathrm{m}$ (Earle et al., 2010), i.e. in the range where volume and surface nucleation are equally important. The size dependence of the freezing rates in Fig. 3 is also consistent with findings by Duft and Leisner (2004), who used the two radii 49 and $19 \mu \mathrm{m}$ in their experiments and concluded that volume nucleation is dominant for those sizes.

\section{Discussion}

The model results indicate that a surface-based freezing mechanism predominates for small droplets. Through Eq. (4), the model considers a surface nucleation event to be a process occurring at the droplet surface (one can think of a process initiated in the surface monolayer of a droplet); however, such an event can also be considered in terms of an ice germ forming in the bulk liquid at a location sufficiently close to the surface that the nucleus comes into contact with the surface before reaching its critical size (Djikaev, 2008; Kay et al., 2003). This view is also consistent with results from molecular dynamics simulations by Vrbka and Jungwirth (2006) who show that nucleation preferentially starts in the subsurface layer. The free energy of formation for such a nucleus attached to the surface (liquid-vapour interface) may be lower than that of a germ forming and remaining completely within the bulk liquid (Djikaev et al., 2002). A lowered free energy of formation would result in an increased nucleation rate coefficient, but this would only apply within this surface layer, a small volume compared to the total volume of the droplet. Our results may be interpreted by using this idea of sub-surface germ initiation followed by attach- ment to the surface. We can express $J_{\mathrm{t}}$, the total rate on a per droplet basis, as:

$J_{\mathrm{t}}=J_{\mathrm{V}} \frac{4 \pi}{3} r^{3}+\tilde{J}_{\mathrm{V}} 4 \pi r^{2} s$

Here $\tilde{J}_{\mathrm{V}}$ is the enhanced volume-specific rate coefficient acting in the surface layer of thickness $s \ll r$. The volume of this surface layer, to a good approximation, is $4 \pi r^{2} s$, and the volume lying below it is much larger and may still be taken to be equal to the whole volume of the droplet. A first approximation of the thickness $s$ may be obtained by using the diameter of a critical germ that would form within the liquid, which is on the order of 2 to $3 \mathrm{~nm}$ in the temperature range $-30^{\circ} \mathrm{C}$ to $-40^{\circ} \mathrm{C}$ (e.g. Pruppacher and Klett, 1997, Figs. 6-7). Here we choose $s=2.5 \mathrm{~nm}$. This thickness may be smaller, however, since reduced free energy of formation may result in a smaller germ size.

The classical formulation for homogeneous freezing, which uses only a volume-specific nucleation coefficient, can be used to describe $\tilde{J}_{\mathrm{V}}$, and hence the surface freezing process. We can modify our expression for $J_{\mathrm{V}}$ (Eq. 2) to account for the lowered free energy of formation of a surface-attached nucleus. If we assume the free energy is lowered by $\Delta W^{*}$ $\left(\Delta W^{*}<0\right)$, then

$\tilde{J}_{\mathrm{V}}=\frac{N_{\mathrm{V}} k T}{h} \exp \left(-\frac{A-B T+\Delta W^{*}}{k T}\right)=J_{\mathrm{V}} \exp \left(-\frac{\Delta W^{*}}{k T}\right)(7)$

Inserting Eq. (7) into Eq. (6) yields

$J_{\mathrm{t}}=J_{\mathrm{V}} \frac{4 \pi}{3} r^{3}\left(1+\frac{3 s}{r} \exp \left(-\frac{\Delta W^{*}}{k T}\right)\right)$

If we define the critical radius again as the radius where both contributions (volume and enhanced surface-layer nucleation) in Eq. (8) are the same, then

$r_{\mathrm{c}}=3 s \exp \left(-\frac{\Delta W^{*}}{k T}\right)$

In addition to the fits with simultaneous volume and surface nucleation using the combined dataset (small, medium and large aerosols; see Sect. 4), we also performed a model fit using Eq. (8) with $J_{\mathrm{V}}$ (i.e. $A_{\mathrm{V}}$ and $B_{\mathrm{V}}$ ) and $\Delta W^{*}$ as adjustable parameters. From this model run, the parameters 
that best fit the results from experiments with small, medium and large aerosols are given in Table 2. The reduction in free energy of formation for a surface-attached nucleus in this case is $\Delta W^{*}=-2.13 \times 10^{-20} \mathrm{~J}$. The quality of fit parameter $\chi$ is almost the same as in our previous fit (using Eq. 4 together with Eqs. 2 and 3). This indicates that the model cannot be used to determine the physical mechanism of surface freezing, i.e. to decide whether the surface nucleation is a pure surface process or an enhanced volume process in a thin shell at the droplets surface; hence, both formulations are equivalent in describing our data. Furthermore, the critical radius $r_{\mathrm{c}}$ given by Eq. (9) is approximately $5 \mu \mathrm{m}$ (between 5.4 and $5.2 \mu \mathrm{m}$ over the range of freezing temperatures for $s=2.5 \mathrm{~nm}$ ), which is within the range determined from the combined volume and surface fit in the previous section.

The fitting procedure is much more sensitive to $\Delta W^{*}$ than to the choice of $s$ because the rate coefficient varies exponentially with $\Delta W^{*}$ and only linearly with $s$ (see Eq. 8). Minimization using a smaller value of $s=1.5 \mathrm{~nm}$ resulted in the same value of $\chi$ and the product $s \exp \left(-\Delta W^{*} /(k T)\right)$ also remained approximately constant $(1.8 \mu \mathrm{m}$ for $s=2.5 \mathrm{~nm}$ and $2.0 \mu \mathrm{m}$ for $s=1.5 \mathrm{~nm}$ at $235.5 \mathrm{~K}$ ). We therefore concluded that fixing the value of $s$ did not significantly influence the results.

If one assumes that the decrease in free energy $\Delta W^{*}$ is due only to different surface energies at the ice-vapour, watervapour and water-ice interfaces (Djikaev et al., 2002), and that the surface area of the critical ice germ, $\Omega_{\mathrm{g}}$, is the same for both a germ forming in the liquid or attached to the droplet surface, then the surface energy, and hence the free energy of formation, of the system with the nucleus attached to the surface is lowered by $\Delta W^{*} \approx\left(\sigma_{\mathrm{i} / \mathrm{v}}-\sigma_{\mathrm{w} / \mathrm{v}}-\sigma_{\mathrm{w} / \mathrm{i}}\right) f \Omega_{\mathrm{g}}$, where $f$ is the fraction of the nucleus surface that is attached to the droplet surface. Thus, we can use our result for $\Delta W^{*}$ to estimate $\sigma_{\mathrm{i} / \mathrm{v}}-\sigma_{\mathrm{w} / \mathrm{v}}-\sigma_{\mathrm{w} / \mathrm{i}}$ (the partial wetting criterion). By assuming a value of $f$ on the order of $15 \%$ and a spherical nucleus with $2.5 \mathrm{~nm}$ diameter, $\sigma_{\mathrm{i} / \mathrm{v}}-\sigma_{\mathrm{w} / \mathrm{v}}-\sigma_{\mathrm{w} / \mathrm{i}} \approx-7 \mathrm{~mJ} \mathrm{~m}^{-2}$ (from our $\Delta W^{*} \approx-6.6 \times k T$ at $235.5 \mathrm{~K}$ ), which is well within the range of uncertainties for reported surface tension values (e.g. Pruppacher and Klett, 1997). Djikaev (2008) reports an estimate of $\Delta W^{*} \approx-5.7 \times k T$ by using available free energies of formation and surface tension data (such that $\sigma_{\mathrm{i} / \mathrm{v}}-\sigma_{\mathrm{w} / \mathrm{v}}-\sigma_{\mathrm{w} / \mathrm{i}}=-5 \mathrm{~mJ} \mathrm{~m}^{-2}$ ). This estimate of $\Delta W^{*}$ is close to our value determined from experiments without assuming values for free energy and surface tensions.

When both volume and surface nucleation processes are considered, the surface contribution to the total nucleation rate $J_{\mathrm{t}}$ can be incorporated into the volume specific rate coefficient to yield a volume specific total nucleation rate coefficient, $J_{\mathrm{V}, \mathrm{t}}$, such that $J_{\mathrm{t}}=J_{\mathrm{V}, \mathrm{t}}(4 \pi / 3) r^{3}$. From either Eq. (4) or (8) it can be seen that $J_{\mathrm{V}, \mathrm{t}}$ is a function of the droplet radius:

$J_{\mathrm{V}, \mathrm{t}}=J_{\mathrm{V}}\left(1+\frac{3 J_{\mathrm{S}}}{r J_{\mathrm{V}}}\right)=J_{\mathrm{V}}\left(1+\frac{3 s}{r} \exp \left(-\frac{\Delta W^{*}}{k T}\right)\right)$
For $r \gg 5 \mu \mathrm{m}$ we obtain $J_{\mathrm{V}, \mathrm{t}} \approx J_{\mathrm{V}}$ because the surface process does not play a significant role for these large droplets. The majority of laboratory studies assume only volume nucleation and hence effectively determine the total rate coefficient, $J_{\mathrm{V}, \mathrm{t}}$, that corresponds to the particle size used in the experiment. The spread observed in the literature data neither confirms nor rules out the possibility of surface nucleation and cannot be reconciled based on the size-dependence of the reported rates because of large systematic uncertainties between data obtained in different studies. Assessing the relative roles of volume and surface nucleation requires experiments with different aerosol sizes under the same experimental conditions, as has been presented here.

According to the above discussion, the results for $J_{\mathrm{V}}$ we presented earlier in Fig. 1 (assuming volume only freezing mechanism) should be the same as our results expressed in terms of $J_{\mathrm{V}, \mathrm{t}}$. This is illustrated in Fig. 4, which, in addition to $J_{\mathrm{V}, \mathrm{t}}$ curves for radii corresponding to our small, medium and large aerosols also shows the nucleation rate coefficient calculated for larger droplets $(r \gg 5 \mu \mathrm{m})$. The radius dependence of the nucleation rate has important implications for climate models. Droplets that are small, for example shortly after their formation, freeze with a significantly increased $J_{\mathrm{V}, \mathrm{t}}$ as compared to larger droplets. For instance, droplets with radius of $1 \mu \mathrm{m}$ have a $J_{\mathrm{V}, \mathrm{t}}$ that is more than 6 times larger than $J_{\mathrm{V}, \mathrm{t}}$ for large droplets. Most current models use purely volume-based nucleation schemes. Using $J_{\mathrm{V}, \mathrm{t}}$ instead of $J_{\mathrm{V}}$ in numerical models will effectively add the factor $1+(3 s / r) \exp \left(-\Delta W^{*} /(k T)\right)$ as a result of including the surface process in addition to volume nucleation.

\section{Conclusions}

Freezing experiments were performed using supercooled liquid water droplets with mean radii of $1.0,1.7$ and $2.9 \mu \mathrm{m}$ (small, medium and large aerosols, respectively). A microphysical model was used to fit our experimental data and determine homogeneous freezing rates. Analysis of the size dependent freezing data confirmed that the classical, volumebased nucleation theory is valid for larger droplets. However, as the droplet size decreases, surface freezing becomes progressively more important. The surface freezing process can be described as volume freezing that occurs sufficiently close to the droplet surface that the critical germ attaches to the surface during its growth, lowering the free energy of formation. Our model results indicate that the free energy of formation of an ice germ attached to the surface of the droplet is lowered by approximately $6.6 \times k T$. Surface and volume nucleation rates become comparable for water droplets with radii of around $5 \mu \mathrm{m}$, with surface rates predominating at smaller sizes. A different formulation assuming a pure surface process with a surface specific rate coefficient, $J_{\mathrm{S}}$, can describe our data equally as well. Thus, we cannot conclude with 
certainty the exact mechanism responsible for enhanced surface nucleation.

The possibility of implementing surface nucleation in models, which currently use only volume nucleation, by adding the factor $\left(1+(3 s / r) \exp \left(-\Delta W^{*} /(k T)\right)\right)$ to $J_{\mathrm{V}}$ has been demonstrated. For example, this increases $J_{\mathrm{V}}$ by a factor of about 6 for aerosols having radii around $1 \mu \mathrm{m}$. We found $\Delta W^{*}=-2.13 \times 10^{-20} \mathrm{~J}$ for $s=2.5 \mathrm{~nm}$ by fitting our data using the microphysical model with Eq. (8).

Acknowledgements. This research was supported by the Natural Sciences and Engineering Research Council of Canada, the Canadian Foundation for Climate and Atmospheric Science and the Ontario Research and Development Challenge Fund. The authors would also like to thank Alex Zasetsky, Brendan Pinto and Monica Harvey for their assistance with the experimental measurements and microphysical model and Wayde Johnson for conducting the computational fluid dynamics analysis of our aerosol flow tube. Some of the runs of the microphysical model, implemented in Matlab (The Mathworks, Inc.), were performed on a server of the Shared Hierarchical Academic Research Computing Network (SHARCNET: www.sharcnet.ca).

Edited by: T. Koop

\section{References}

Bohren, C. F. and Huffman, D. R.: Absorption and Scattering of Light by Small Particles, Wiley-Interscience, New York, USA, 477 ff., 1983.

Cantrell, W. and Heymsfield, A.: Production of ice in tropospheric clouds - A review, B. Am. Meteorol. Soc., 86, 795-807, doi:10.1175/BAMS-86-6-795, 2005.

Cox, S. K.: Cirrus Clouds and the Climate, J. Atmos. Sci., 28, 1513-1515, 1971.

DeMott, P. J. and Rogers, D. C.: Freezing nucleation rates of dilutesolution droplets measured between -30 and $-40^{\circ} \mathrm{C}$ in laboratory simulations of natural clouds, J. Atmos. Sci., 47, 10561064, 1990.

DeMott, P. J., Meyers, M. P., and Cotton, W. R.: Parameterization and impact of ice initiation processes relevant to numericalmodel simulations of cirrus clouds, J. Atmos. Sci., 51, 77-90, 1994.

DeMott, P. J., Rogers, D. C., and Kreidenweis, S. M.: The susceptibility of ice formation in upper tropospheric clouds to insoluble aerosol components, J. Geophys. Res.-Atmos., 102, 1957519584, doi:10.1029/97JD-01138, 1997.

Djikaev, Y.: Effect of the surface-stimulated mode on the kinetics of homogeneous crystal nucleation in droplets, J. Phys. Chem. A, 112, 6592-6600, doi:10.1021/jp021044s, 2008.

Djikaev, Y. S., Tabazadeh, A., Hamill, P., and Reiss, H.: Thermodynamic conditions for the surface-stimulated crystallization of atmospheric droplets, J. Phys. Chem. A, 106, 10247-10253, doi:10.1021/jp021044s, 2002.

Duft, D. and Leisner, T.: Laboratory evidence for volumedominated nucleation of ice in supercooled water microdroplets, Atmos. Chem. Phys., 4, 1997-2000, doi:10.5194/acp-4-19972004, 2004.
Earle, M. E., Kuhn, T., Khalizov, A. F., and Sloan, J. J.: Volume Nucleation Rates for Homogeneous Freezing in Supercooled Water Microdroplets: Results from a Combined Experimental and Modelling Approach, Atmos. Chem. Phys., 10, 7945-7961, doi:10.5194/acp-10-7945-2010, 2010.

Heymsfield, A., Miloshevich, L., Schmitt, C., Bansemer, A., Twohy, C., Poellot, M., Fridlind, A., and Gerber, H.: Homogeneous ice nucleation in subtropical and tropical convection and its influence on cirrus anvil microphysics, J. Atmos. Sci., 62, 4164, 2005.

Heymsfield, A. J. and Miloshevich, L. M.: Homogeneous ice nucleation and supercooled liquid water in orographic wave clouds, J. Atmos. Sci., 50, 2335-2353, 1993.

Heymsfield, A. J. and Sabin, R. M.: Cirrus Crystal Nucleation by Homogeneous Freezing of Solution Droplets, J. Atmos. Sci., 46, 2252-2264, 1989.

Jensen, E., Toon, O., Westphal, D., Kinne, S., and Heymsfield, A.: Microphysical modeling of cirrus. 2. Sensitivity studies, J. Geophys. Res.-Atmos., 99, 10443-10454, doi:10.1029/94JD-00226, 1994.

Jensen, E., Toon, O., Tabazadeh, A., Sachse, G., Anderson, B., Chan, K., Twohy, C., Gandrud, B., Aulenbach, S., Heymsfield, A., Hallett, J., and Gary, B.: Ice nucleation processes in upper tropospheric wave-clouds observed during SUCCESS, Geophys. Res. Lett., 25, 1363-1366, doi:10.1029/98GL-00299, 1998.

Jensen, E. J. and Toon, O. B.: Ice nucleation in the upper troposphere - sensitivity to aerosol number density, temperature, and cooling rate, Geophys. Res. Lett., 21, 2019-2022, doi:10.1029/94GL-01287, 1994.

Kay, J. E., Tsemekhman, V., Larson, B., Baker, M., and Swanson, B.: Comment on evidence for surface-initiated homogeneous nucleation, Atmos. Chem. Phys., 3, 1439-1443, doi:10.5194/acp-31439-2003, 2003.

Khalizov, A. F., Earle, M. E., Johnson, W. J. W., Stubley, G. D., and Sloan, J. J.: Development and characterization of a laminar aerosol flow tube, Rev. Sci. Instrum., 77, 033102 , doi:10.1063/1.2175958, 2006.

Krämer, B., Hübner, O., Vortisch, H., Wöste, L., Leisner, T., Schwell, M., Ruhl, E., and Baumgärtel, H.: Homogeneous nucleation rates of supercooled water measured in single levitated microdroplets, J. Chem. Phys., 111, 6521-6527, 1999.

Lawson, R., Baker, B., Pilson, B., and Mo, Q.: In situ observations of the microphysical properties of wave, cirrus, and anvil clouds. Part II: Cirrus clouds, J. Atmos. Sci., 63, 3186-3203, 2006.

Liou, K.: Influence of cirrus clouds on weather and climate processes - A global perspective, Mon. Weather. Rev., 114, 11671199, 1986.

Pruppacher, H. R. and Klett, J. D.: Microphysics of Clouds and Precipitation, Kluwer Academic Publishers, Dordrecht, The Netherlands, 2nd edn., 1997.

Rogers, D., DeMott, P., Kreidenweis, S., and Chen, Y.: Measurements of ice nucleating aerosols during SUCCESS, Geophys. Res. Lett., 25, 1383-1386, doi:10.1029/97GL-03478, 1998.

Rosenfeld, D. and Woodley, W.: Deep convective clouds with sustained supercooled liquid water down to $-37.5^{\circ} \mathrm{C}$, Nature, 405 , 440-442, 2000.

Sassen, K., Liou, K. N., Kinne, S., and Griffin, M.: Highly Super- 
cooled Cirrus Cloud Water: Confirmation and Climatic Implications, Science, 227, 411-413, 1985.

Sigurbjörnsson, Ó. F. and Signorell, R.: Volume versus surface nucleation in freezing aerosols, Phys. Rev. E, 77, doi:10.1103/PhysRevE.77.051601, 2008.

Stöckel, P., Weidinger, I., Baumgärtel, H., and Leisner, T.: Rates of homogeneous ice nucleation in levitated $\mathrm{H}_{2} \mathrm{O}$ and $\mathrm{D}_{2} \mathrm{O}$ droplets, J. Phys. Chem. A, 109, 2540-2546, doi:10.1021/jp047665y, 2005.

Tabazadeh, A., Djikaev, Y. S., and Reiss, H.: Surface crystallization of supercooled water in clouds, Proc. Natl. Acad. Sci., 99, 15873-15878, doi:10.1073/pnas.252640699, 2002.

Turnbull, D. and Fisher, J.: Rate of nucleation in condensed systems, J. Chem. Phys., 17, 71-73, 1949.

Vrbka, L. and Jungwirth, P.: Homogeneous freezing of water starts in the subsurface, J. Phys. Chem. B, 37, 18126-18129, doi:10.1021/jp064021c, 2006.
Wood, S. E., Baker, M. B., and Swanson, B. D.: Instrument for studies of homogeneous and heterogeneous ice nucleation in freefalling supercooled water droplets, Rev. Sci. Instrum., 73, 39883996, 2002.

Zasetsky, A., Khalizov, A., Earle, M., and Sloan, J.: Frequency dependent complex refractive indices of supercooled liquid water and ice determined from aerosol extinction spectra, J. Phys. Chem. A, 109, 2760-2764, doi:10.1021/jp044823c, 2005.

Zasetsky, A., Earle, M., Cosic, B., Schiwon, R., Grishin, I., McPhail, R., Pancescu, R., Najera, J., Khalizov, A., Cook, K., and Sloan, J.: Retrieval of aerosol physical and chemical properties from mid-infrared extinction spectra, J. Quant. Spectrosc. Ra., 107, 294-305, doi:10.1016/j.jqsrt.2007.02.016, 2007.

Zasetsky, A. Y., Khalizov, A. F., and Sloan, J. J.: Characterization of atmospheric aerosols from infrared measurements: simulations, testing, and applications, Appl. Opt., 43, 5503-5511, 2004. 